\title{
NEOLIBERALISMO Y NEOGERENCIA PÚBLICA ${ }^{1}$
}

\author{
Omar Guerrero ${ }^{2}$
}

http://dx.doi.org/10.1590/1413-2311.246.90269

\begin{abstract}
RESUMEN
El artículo se propone examinar a la nueva gerencia pública en su totalidad histórica, como un fenómeno emanado del surgimiento del neoliberalismo en el siglo XX. Particularmente examina el papel de esa gerencia como instrumento de las reformas del Estado planteadas por el neoliberalismo entre 1990 y 2010, destacando sus aspectos doctrinarios e instrumentales. Enfatiza, en fin, la incorporación de conceptos económicos introducidos por la nueva gerencia pública en el campo de estudio de la administración pública.
\end{abstract}

Palabras clave: Neoliberalismo. Nueva gerencia pública. Administración pública. Privatización y mercado.

\begin{abstract}
The article proposes to examine the new public management in its historical totality, as a phenomenon emanated from the emergence of neoliberalism in the 20th century. In particular, it examines the role of that management as an instrument of the state reforms proposed by neoliberalism between 1990 and 2010, highlighting its doctrinal and instrumental aspects. In short, emphasizes the incorporation of economic concepts introduced by the new public management in the field of study of public administration.

\footnotetext{
${ }^{11}$ Recebido em 12/02/2019; aprovado em 15/04/2019.

${ }^{2}$ Universidad Nacional Autónoma de México - Facultad de Ciencias Políticas y Sociales; Ciudad de México (México) - http://orcid.org/0000-0001-9830-6355. E-mail: omarguerrer@gmail.com.
} 
Keywords: Neoliberalism. New public management. Public administration. Privatization and market.

\section{INTRODUCCIÓN}

Este artículo versa sobre la nueva gerencia pública, un fenómeno social suscitado entre las décadas de 1990 y 2010, si bien su origen se remonta a 1966 y todavía se dejan sentir algunos de sus afectos. Se trata, pues, de un fenómeno histórico que puede ser estudiado como totalidad social en un tiempo dado.

Su rasgo esencial es que se constituyó como una creatura del neoliberalismo, en la administración pública. La nueva gerencia pública fue, en buena medida, su mejor instrumental para llevar a cabo las reformas económicas que se propuso implementar en escala planetaria.

El presente trabajo está principalmente integrado por el capítulo 5 de nuestro libro $L a$ Nueva Gerencia Pública, publicado en México por Fontamara en 2004.

\section{EL NEOLIBERALISMO}

El neoliberalismo es una doctrina cuya fecha y lugar de nacimiento son sabidos: nació en París, en el año de 1938.

\subsection{LA FUNDACIÓN}

Fue en ese "entonces que los economistas de todos los países opinaron que había llegado el momento de oponer un liberalismo renovado y mejorado a un socialismo cuya incapacidad acababa de demostrarse" (BAUDIN, 1956, p. 38). La resurrección liberal fue trascendental para sus promotores por cuanto significó la unificación de fuerzas intelectuales, que hasta entonces se habían mantenido dispersas. En efecto, el momento de la unión ocurrió en octubre 26-30 de 1938, con motivo de un coloquio organizado en París por motivo de la publicación del libro de Walter Lippmann, The Good Society (La Buena Sociedad) (LIPPMANN, 1937, pp. VII-VIII) recién traducido al francés (LIPPMANN, 1938). Pocos escritos pueden exaltar un grado de repercusión tan intenso, como la obra de Lippmann, tocando "las palmas" a la versión francesa por constituir su presentación el precipitante intelectual de las discusiones parisinas. No sin razón, el texto de Lippmann fue considerado 
por André Maurois como uno de los lados del triángulo bibliográfico que concibió al neoliberalismo. En efecto, el intelectual francés justiprecia El Socialismo de von Mises y Las Místicas Económicas de Louis Rougier, junto con La Buena Sociedad, como manantial que dio vida al liberalismo renovado (LIPPMANN, 1937, p. 10). Dicho coloquio congregó, entre otros pensadores conspicuos, a Ludwig von Mises y Friedrich Hayek.

\subsection{LA DOCTRINA NEOLIBERAL}

El Coloquio Lippmann mostró que los liberales de entonces estaban unidos por fuertes lazos de asentimiento en temas centrales, pero asimismo, que los separaban apreciaciones relevantes. Uno de los participantes más destacados, Louis Baudin, declaró expresamente su disentimiento sobre el nombre de la doctrina, como "neoliberalismo", porque la libertad no fue la finalidad exclusiva que la reunión se propuso alcanzar; y si el laissez-faire, laissezpasser fue unánimemente rechazado, tampoco le pareció correcto tomar la etiqueta de un sistema caduco como el liberalismo, incluso con la adición de "neo". "No obstante, se decidió así y el neoliberalismo entró en la historia" (BAUDIN, 1956, p. 146).

\subsubsection{La doctrina}

No está por demás insistir en el hecho de que el nombre neoliberalismo no sólo fue escogido por quienes lo profesan como su doctrina, sino que así lo han utilizado desde entonces. Incluso, Baudin mismo gozó el crédito de ser uno de sus profesantes, tal como lo hace constar el autor del prólogo de una de sus obras, quien señala con énfasis que "sus innumerables obras, algunas de ellas en varias ediciones, han marcado la pauta en la moderna concepción del neoliberalismo (...) Baudin es un representante clásico de la libertad económica. Como uno de los fundadores del neoliberalismo, verdadera solución a los problemas de la economía moderna (...)” (NAVARRO, VÁZQUEZ, AGUSTÍN, 1956).

\subsection{LA AGENDA}

Louis Baudin nos relatará puntualmente la agenda de la reunión de París:

El acta de nacimiento del neoliberalismo consiste en una agenda que comprende cuatro puntos esenciales:

1. El nuevo liberalismo admite que sólo el mecanismo de los precios, funcionando en mercados libres, permite obtener una utilización 
óptima de los medios de producción y conducir a la satisfacción máxima de los deseos humanos.

2. Al Estado incumbe la responsabilidad de determinar el régimen jurídico que sirva de marco al libre desarrollo económico así concebido.

3. Otros fines sociales pueden ser substituídos por los objetivos económicos enunciados más arriba.

4. Una parte de la renta nacional puede ser, con ese objeto, substraída al consumo, pero con la condición de que la transferencia se haga a 'plena luz' y sea 'conscientemente consentida'.

A esos cuatro puntos conciernen, pues, respectivamente, la clave del sistema, su marco, sus objetos, sus medios de aplicación” (BAUDIN, 1956, p. 150).

El Coloquio Lippmann finalizó con la redacción de una agenda que sirviera de cauce al movimiento neoliberal, el cual, como puede observarse hasta nuestros días, se ha seguido al pie de la letra.

\subsection{NACIMIENTO DE LA NUEVA GERENCIA PÚBLICA}

La doctrina y la agenda del neoliberalismo reseñados no abordaron directamente a la administración pública, si bien, trataron con alguna extensión el tema del Estado. Este silencio epistemológico fue roto a mediados de la década de 1960, cuando Octave Gelinier produjo un primer planteamiento neoliberal sobre la administración pública (GELINIER, 1966), estableciendo las bases de lo que posteriormente sería la nueva gerencia pública. Una década después fue publicado un libro muy singular en el cual el neoliberalismo redobla sus primeros pasos en el ámbito de la administración pública, y se nombra precisamente a su proyecto: nueva gestión pública (nouvelle gestion publique) (MASSENET, 1975). Su autor es Michel Massenet. Cabe puntualizar no sólo la particularidad del título de la obra, sino también su contenido y proposiciones, así como el tiempo en que fue publicado, es decir, la época cuando el Estado de bienestar gozaba de plena salud. Además, el subtítulo propone un "Estado sin burocracia", para poner en su lugar un régimen mercantil donde domina la competencia y el cliente es quien dicta los lineamientos del proceso económico. Este libro singular proveyó de un material insólito, difícil de comprender en ese entonces, y ciertamente insatisfactorio para los círculos académicos habituados al estudio de la burocracia, la 
jerarquía y la autoridad, que es propiamente el locus usual de los administradores públicos. Hay que destacar que no se trata de un hecho aislado, pues la obra de Gelinier sirvió de inspiración a Massenet, y le proveyó de energía para que la nueva doctrina prosperara y se diera conocer.

Un tercer paso trascendental fue la publicación en 1980 de un texto francés, sin conexión bibliográfica con los anteriores, pero sí con un parentesco conceptual íntimo. Se trata de la obra de Romain Laufer y Alain Burlaud, quienes refuerzan el esquema esencial de la nueva gerencia pública en su libro Gerencia Pública: Gestión y Legitimidad (Management Public: Gestion y Legitimite) (LAUFER, BURLAUD, 1980), toda vez que es posible que ellos fueran quienes optaran primigeniamente por el término "gerencia pública", en lugar de "gestión pública".

Las propuestas de la nueva gestión pública en la época de Gelinier y Massenet, eran desajustadas, exóticas e irrealizables. Cuán lejos se estaba entonces de anticipar lo que ocurriría a finales de la década de 1980, cuando la "nueva gestión pública" emergió rebautizada como "nueva gerencia pública". Las ideas, no cabe duda, tienen capacidad de germinación, desarrollo, supervivencia y adaptación, que con frecuencia no alcanzamos a comprender plenamente en un momento que se juzga como actual y entonces vivido. Fue la "necesidad" maquiaveliana, que da forma a las condiciones sociales, la que creó la posibilidad a través de la cual el neoliberalismo, una doctrina en maduración, desarrollara un proyecto dotado con instrumentos de acción efectivos. El ayuno neoliberal de un programa dotado con efectividad, evidente desde finales de la década de 1930, prosperó a partir de los decenios de 1960 y 1970 cuando germinó la "nueva gestión pública" y demostró que la doctrina de los nuevos liberales podría ser implementable. El ensayo, a modo de tanteo de posibilidades, fue exitoso, pues década y media después la "nueva gerencia pública" (new public management) vino a la vida.

En efecto, el origen, desarrollo y declinación de la nueva gerencia pública es inexplicable sin la consideración de sus vínculos parentales con el neoliberalismo. Esto obedece a las siguientes consideraciones: en primer lugar, el neoliberalismo emergió a finales de la década de 1930 como una reivindicación del liberalismo, pero sustentado en nuevas fuentes y como una réplica al avance planetario del socialismo, y particularmente por motivo del progreso del intervencionismo en las economías occidentales; sin embargo, emergió a la vida carente de un instrumental que viabilizara su programa de acción. En segundo lugar, como lo adelantamos, la nueva gerencia pública surge a la vida a mediados de la década de 1960, bajo el sustento de un incipiente número de ideas basadas en la economía de mercado, 
la competencia y la orientación al cliente, pero con la forma de un recetario práctico apenas sustentado doctrinalmente. De modo que el neoliberalismo adquiere consistencia programática a través del desarrollo de la nueva gerencia pública; y la nueva gerencia pública obtiene un cuerpo de doctrina por medio del neoliberalismo para sustentar su desarrollo.

\section{LA NUEVA GERENCIA PÚBLICA}

Del mismo modo como ha ocurrido en la empresas privada y el derecho administrativo, entre otras disciplinas sociales, las transformaciones contemporáneas de la administración pública han demandado un esfuerzo de conocimiento sin paralelo. Ella, como disciplina científica, inveteradamente se ha visto sujeta al flujo y reflujo de sus fronteras, sea con referencia a la gerencia privada, sea con el derecho administrativo. Buena parte de las lides que ha logrado en pro de su independencia han sido ganadas a sus dos disciplinas hermanas. Asimismo, algunas décadas atrás, enfrentó un desafío inédito, entonces planteado por la economía política, de la cual se había terminado de divorciar por mutuo acuerdo hacía un siglo. El problema radica en una problematización por la cual los defectos de la administración pública, se supone, son corregibles por medio del uso de las conceptuaciones de la economía. Empero, no se debe deducir que los consejos vendrán desde la óptica keynesiana, ni mucho menos del nuevo mercantilismo. Obviamente, ni pensar en el ideario del Estado providencia. El remedio propuesto proviene de la economía neoclásica, especialmente desde el neoliberalismo engendrado en la escuela austriaca y la opción pública estadounidense. La forma que personifica el neoliberalismo en administración pública es la nueva gerencia pública.

\subsection{UNA IMAGEN BÁSICA}

La difusión planetaria de la nueva gerencia pública parte del siguiente diagnóstico: hacia finales de la década de 1980, crisis recurrentes en el espacio económico de dentro y de fuera del Estado parecieron insalvables. Paralelamente, las sociedades comenzaron a naufragar como víctimas de desarreglos institucionales, en tanto que la esfera política se descomponía al tenor de su incapacidad de aliviar los males de la sociedad. Yendo más allá de los planteamientos primigenios de Gelinier y Massenet, fue entonces que se convocaron antiguos planteamientos de la economía neoclásica formulados por economistas neoliberales conspicuos -especialmente Ludwig von Mises, Friedrich Hayek, Milton Friedman y James 
Buchanan-, cuyas prédicas invocaron el retorno a la vida sencilla del intercambio mercantil, evocándose la filosofía económica elemental de Adam Smith y el rigor existencial de los primeros angloamericanos. Particularmente, Friedman propuso reconsiderar a una sociedad mercantil formada por productores y consumidores cuyo intercambio sería temporal y en el momento mismo de la transacción, para luego, en su aislamiento individual dar rienda suelta a su privacidad (FRIEDMAN; FRIEDMAN R., 1979, pp. 1-7).

Ante una sencilla sociedad natural como la concebida por la economía neoclásica, el Estado administrativo resulta no sólo artificial, sino estorboso, y nada mejor que procurar su reforma desde la raíz. Consecuentemente, un modelo gerencial emergente fue propuesto como sustituto de una administración pública que se concibe como agotada, al igual que el Estado del que deriva. Sin embargo, esta visión propone una brecha antinómica entre la administración pública y la nueva gerencia pública. Bajo su auspicio se configuran pares opuestos, una dicotomía que separa a la "tradicional", "weberiana" y "burocrática" administración pública, de un nuevo paradigma gerencial considerado como "ágil”, "eficiente" y "moderno".

La nueva gerencia pública propone una renovada esencia empresarial dentro del gobierno, en reemplazo de la presumiblemente agotada naturaleza burocrática. Esa gerencia es el resultado de un proceso constituido por dos etapas de privatización de los espacios públicos. La primera fase entraña la exoprivatización del Estado y consiste en el procedimiento por el cual la administración pública trasfiere la producción de bienes y servicios a la administración privada, moviendo al Estado hacia fuera del mercado. La segunda etapa consiste en la endoprivatización del Estado, es decir, la sustitución la administración pública de los asuntos nacionales, por la idea, la metodología y la técnica de la gerencia privada, moviendo al mercado hacia dentro del Estado. En la exoprivatización la gerencia pública funge como el sujeto de la transacción de cosas hacia el mercado; en la endoprivatización esa gerencia se convierte el objeto de la transacción mercantil. En la primera etapa la gerencia pública es el mercader, en la segunda fase es la mercancía. En tanto la exoprivatización afecta al qué de la administración pública, la endoprivatización lo hace con el cómo.

El mero enunciado de las ideas precedentes debería concitar un fácil acuerdo, acerca de que los materiales que constituyen la sustancia de la nueva gerencia pública son neoliberales. Empero, esta presunción resulta inaceptable y antipática para algunos, así como temeraria para otros. Quizá estas incomodidades obedecen al sentir general de que el neoliberalismo ha sido entre nuestros pueblos un caudal de pauperización, una fuente de 
descomposición social y un ácido para la convivencia política. Es cierto que nuestros pueblos no eran del todo felices antes de su paso triunfante desde principios de la década de 1980, pero ciertamente son menos felices ahora. Esto y otros problemas más hacen imperativo entender con profundidad el significado de la nueva gerencia pública como una creatura neoliberal, destacándose su esencia económica neoclásica.

Quizá la identificación primigenia del fenómeno gerencial dominante que ha nutrido las reformas administrativas en el cosmos anglosajón, se deba a la pluma de Christopher Hood (1991, pp 5-6). Este fenómeno gerencial está originalmente integrado por dos corrientes de ideas. La primera la constituye la opción pública, la teoría de los costos de transacción y la teoría del principal-agente (AUCOIN, 1996, pp. 491-515). Sus contribuciones a la reforma administrativa son las nociones de costeabilidad, opción del usuario y transparencia. La segunda corriente fluye desde el viejo gerencialismo de negocios sobreviviente en el sector público, un remanente ya borroso del movimiento de la "gerencia científica" de Frederick Taylor y Henri Fayol, cuyos aportes son el manejo profesional y el poder discrecional para lograr resultados, también conocida como libertad de manejo.

En tanto la opción pública provee la idea de primacía del gobierno representativo sobre la burocracia, el gerencialismo brinda el sentido del imperio de la gestión sobre los procesos burocráticos (AUCOIN, 1996). La teoría del principal-agente se adentra en la organización administrativa para describir la relación entre un ministro y un subordinado. Plantea que el ministro cuenta con autoridad suficiente para realizar sus tareas, por cuanto principal es su estatuto, pero que depende de sus subordinados, asistentes y consejeros. La relación principal-agente obedece al límite de tiempo del primero para hacer policy, así como su desconocimiento sobre las materias de esa hechura y de los recursos de los que disponen quienes forman su equipo (BARZELAY, 2000, pp. 197-198).

La nueva gerencia pública constituye un fenómeno de alta complejidad. Ello obedece, en primer lugar, a que a su seno han arribado una variedad de ingredientes, los cuales, aunque son de la misma naturaleza, o están emparentados íntimamente, tienen personalidad propia y autonomía relativa. Como lo adelantamos, en primer lugar destaca la economía neoclásica donde repuntan como aspectos diversos la escuela austriaca y la opción pública. La presencia de la primera es observable principalmente a través de la obra de Ludwig von Mises y Friedrich Hayek. En la segunda son muy visibles James Buchanan, Gordon Tullock, Vincent Ostrom y William Niskaken. También está presente el pensamiento administrativo de Peter Drucker, y una cantidad apreciable de conceptos sobrevivientes de la administración pública que han sido rebautizados. 
Esta combinación no ha producido un ente parecido a Frankenstein, como pudiera pensarse, sino a un ser mutante de difícil conceptuación, y de perfiles móviles y perpetuamente acomodables. Dentro de esa masa de ideas, conceptos, propósitos, valores e intereses, se pueden apreciar ocho conceptos relativamente estables y definitorios de la nueva gerencia pública.

En primer lugar se pueden percibir los conceptos fundacionales, el caldo primordial en el que engendró esa gerencia, a saber: Orientación al cliente; Privatización; Mercado; Competencia.

Después es observable el aparato de sistematización de la reforma neogerencial del sector público, que estructura una nueva base organizativa y operacional. Los conceptos son: Enfoque empresarial-gerencial; Gerencia por objetivos y resultados; Agenciación.

Finalmente emerge el móvil que animó a la nueva gerencia pública, que no es otro que: Reducir costos.

\subsection{CONCEPTOS FUNDACIONALES}

La cuarteta conceptual alrededor de la cual está configurada la nueva gerencia pública, es la orientación al cliente, la privatización, el mercado y la competencia.

\subsubsection{Orientación al cliente}

Uno de los conceptos que mejor han permitido identificar los fundamentos neoliberales de la nueva gerencia pública, es la soberanía del consumidor. La idea parte del hecho de que estando los ciudadanos influidos principalmente por el disfrute de bienes tutelares como la educación, la salud y otros servicios brindados por el Estado benefactor, ha emergido el problema de cómo financiar un enorme gasto con cargo a los ingresos del erario público (OECD, 1987). La solución se materializó en la orientación hacia el cliente, una versión actualizada de la idea de la soberanía del consumidor formulada originalmente por Ludwig von Mises.

Propósitos esencial de las reformas neogerenciales es hacer que la administración pública esté en contacto con los clientes y así establecer un mercado competitivo en su seno. Como se supone que la definición de los objetivos de la administración pública suele ser opaca, su capacidad de respuesta (responsividad) requiere un estilo administrativo distinto. Es decir, un manejo responsivo que capte la capacidad que tiene cada cliente para acceder a las prestaciones que tienen derecho a esperar, pues se ha desarrollado una sutil división entre 
"usuarios de los servicios" y "consumidores de los servicios". Estos últimos, en contraste con aquellos otros, no son concebidos como recipientes pasivos de servicios, sino como clientes activos.

La idea de cliente sustituye a la de ciudadano, provocando que como contribuyentes informados conozcan el nivel de eficiencia de los servicios públicos a través del pago de sus impuestos (KAUL, 1997, pp. 15-16). Por tal motivo, los resultados electorales estarán determinados más por la satisfacción o insatisfacción con los servicios públicos, que por la politización, las campañas políticas y una opinión pública consciente. Tal es el amplio propósito de la Carta Ciudadana del Reino Unido, destinada a los clientes públicos y que contiene los estándares de los servicios gubernamentales.

Una de las nociones más ligadas a la soberanía del consumidor es el "empoderamiento" del cliente. El término tiene hondas raíces en la cultura política occidental, donde significa lo que un locutor o escritor desea transmitir cuando externa su opinión. Significa sencillamente la existencia de un gobierno democrático y de organizaciones abiertas a la participación de sus miembros (PETERS; PIERRE, 2000, pp. 911 y 24-25). Sin embargo, en las reformas neogerenciales el "empoderamiento" ha tendido a referir meramente la condición de cliente y evocar la soberanía del consumidor, pues su despliegue obedece a las críticas hechas a un gobierno opaco, remoto e infranqueble. Siendo soberano, el consumidor asume el control de su vida y su destino, y de tal modo hace trasparente y próximo a su gobierno.

Como derivación de la orientación al cliente, que entraña el mejoramiento de la información de sus preferencias en el mercado, la administración pública sufre alteraciones significativas para tal efecto: las grandes organizaciones que suministran los servicios públicos se desagregan en pequeñas agencias operativas, toda vez que son extintos los monopolios gubernamentales (HOOD, 1987, pp. 145-170). Siendo esas agencias las receptoras directas de las preferencias clientelares, junto con ellas se establecen pagos directos al usuario que tienden a sustituir a los impuestos.

\subsubsection{Privatización}

El nuevo paradigma ha constitu+ido un esfuerzo hacia la privatización de los estilos de la práctica gerencial, que demanda la adopción de las herramientas provenientes del sector privado por parte del sector público (HOOD, 1991, pp. 5-6). La nueva gerencia pública se mueve hacia el aumento de la competencia en el sector público, porque la "rivalidad" es la clave de la disminución de costos y mejores estándares. Por lo tanto, se esfuerza por 
disminuir o revertir el crecimiento del gobierno con base en un cambio hacia la privatización o cuasi-privatización, con un renovado énfasis en la subsidiariedad en la provisión de servicios (HOOD, 1991, p. 3).

La privatización entraña una propuesta para que la administración privada juegue un papel pedagógico en provecho de la administración pública, cuyas lecciones significan inherentemente un buen manejo. Simplemente este último es la aplicación de la sabiduría del manejo convencional y las técnicas concomitantes al sector público, unidas a la planeación, la organización y el control. La aplicación del buen manejo produce "organizaciones excelentes", es decir, aquellas organizaciones que no tienen solamente un desempeño con altos niveles de rentabilidad, sino que son también innovadoras y proveen seguridad de empleo, así como valiosos productos y servicios al consumidor (DUNCAN; GINTER; CAPPER, 1991, p. 230). Consiguientemente, la administración pública podría ser una organización excelente si aprende cuatro lecciones dictadas por la administración de negocios: enfocarse en el desarrollo y preservación de una visión empresarial; practicar un liderazgo efectivo y desechar la dirección tradicional; fomentar la innovación en todos los niveles de la organización; y mejorar las relaciones con los clientes externos (DUNCAN; GINTER; CAPPER, 1991, p. 230).

Conforme la noción de un Estado administrativo rescatable, al cual se le debe dar una infusión sustanciosa de racionalidad, el modelo de nueva gerencia pública se propone reemplazar exitosamente al viejo paradigma vigente de la administración pública. Esta idea es el resultado de la combinación de la perspectiva gerencial y una nueva apreciación del sector público, la cual descansa en la idea de establecer un mercado interior y endoprivatizar los procesos de gestión referentes a la provisión de bienes y servicios públicos, y el manejo de las empresas gubernamentales. Por consiguiente, su éxito potencial está basado en las respuestas positivas a las demandas de efectividad del sector público.

Ese supuesto tiene su raíz en la clausura de la fase de la exoprivatización y la inauguración de la etapa de la endoprivatización, tal como se puede visualizar en el siguiente pasaje: "dado que todas las actividades del gobierno difícilmente pueden ser transferidas al sector privado, la próxima mejor solución es transferir la orcaneta de los negocios a las operaciones gubernamentales. Sin embargo, la gerencia privada es diferente de la administración pública: el primero deriva de las operaciones comerciales y significa traer un nuevo espíritu y un nuevo vocabulario, así como la proliferación de las técnicas del manejo. Esto también significa 'desburocratizar' las operaciones del gobierno y reducirlas substancialmente" (SAVOIE, 1995, p. 113). 
El modelo neogerencial, en suma, es propuesto como conciliable en general con la teoría de la administración pública, pero polemiza sin descanso con un paradigma específico de dicha administración al que llama "weberiano". El modelo weberiano que personifica la esencia del Estado administrativo es observado como un paradigma racional basado en la planeación, así como una organización administrativa jerárquica, que deben ser reemplazados por un esquema de descentralización y orientación hacia los resultados, así como la introducción de mercados internos dentro de los gobiernos.

\subsubsection{Mercado}

La nueva gerencia pública establece mercados interiores en el seno de la administración pública, y utiliza la contratación para fomentar la competencia. Esta fórmula se extiende a la administración de personal donde se ha impuesto el sistema de contratos (BOYLE, 1992-1993, pp. 333-346), y así allana el paso al servicio civil para incorporar candidatos del sector privado en su entraña (GRAY; JENKINS, 1995, p. 87).

La visión neogerencial refleja el crecimiento sobresaliente de los valores del mercado en el sector público. Este enfoque tiene su base en el modelo de conducción del mercado dentro del sector público, y aunque la provisión dicho sector, la forma en que se hace lo es, porque hay un estilo diferente de organización y liderazgo que está fundado en el desarrollo de un mercado interno. El cambio consiste en mudar algunas funciones tradicionales en el sector público y establecer en su interior los elementos del mercado (LANE, 1994, p. 140). Ese cambio está representado por la gerencia, en lugar de la asignación pública de recursos; por la privatización, en lugar de las empresas públicas; por esquemas de incentivos de competitividad, en lugar de la distribución de impuestos; por la desregulación, en lugar de la regulación pública; y por la economía neoclásica, en lugar de la hechura de policy macroeconómica.

En contraste con el Reino Unido y los países de la Commonwealth, en los Estados Unidos de América la nueva gerencia pública intentó deslindarse del modelo de privatización aludiendo que la perspectiva del mercado no constituye una teoría única, sino un conjunto de estrategias alternativas que pueden tener un conflicto entre sí. Es más, allí el enfoque gerencial es considerado como diferente al modelo de privatización (OSBORNE; GAEBLER, p. 45). Para dos pensadores neogerenciales, David Osborne y Ted Gaebler, la privatización es una de las flechas del carcaj del gobierno, pero privatizar no es la solución. Incluso, critican a quienes defienden a la privatización por razones ideológicas y sostienen que la empresa privada es inherentemente superior al gobierno. 
Esta imagen, ciertamente torcida por desconocer la factura privatizadora de la nueva gerencia pública, constituye un apartamiento ideológico por la incomodidad que algunos gobiernos post-privatizadores tienen cuando todavía conservan proles de esas policies mercantiles. Empero, se trata de un cambio de foco de la privatización, la cual, de actuar originalmente hacia afuera, retorna y se introduce en los procesos de gestión pública para establecer un mercado interior, pues su objeto es explotar a los sectores públicos de los países que tienen una economía avanzada, donde el tamaño de la asignación de recursos públicos es substancial y cuentan aún con empresas públicas. En efecto, la nueva gerencia pública es un intento de reforma del sector público desde dentro, en tanto que la privatización implica que las funciones son segregadas del sector público (LANE, 1994, p. 142). La privatización favorece el mercado externo, en tanto que el modelo neogerencial auspicia el mercado interno. Sencillamente la privatización ha cambiado de lugar, pues ha pasado de fuera, hacia adentro. De aquí nuestra idea de diferenciar, como lo hicimos saber, entre exoprivatización y endoprivatización.

Uno de los efectos preponderantes del imperio del mercado es el establecimiento de sus mecanismos dentro de la administración pública, entre los que destacan la contratación por fuera (contracting out), los cargos al usuario y los vales (vouchers) (ORMOND; LÖFFLER, 1998, pp. 7-9). La contratación por fuera es el traspaso de la provisión de servicios al sector privado, en tanto que el cargo a los usuarios es el pago directo de los servicios públicos que realizan para hacerles conocer inmediatamente sus costos y beneficios. Este cargo constituye una de las manifestaciones más nítidas de la orientación al cliente, toda vez que entraña mecanismos típicos de mercado y competencia. Por su parte, los vouchers constituyen un régimen a través del cual el cliente adquiere el derecho a un bien o servicio que él puede cubrir a los proveedores, y que reembolsan en moneda o en su equivalente desde un fondo destinado para tal efecto. Se trata de vales a nombre de personas específicas que reciben un servicio concreto de una lista de proveedores, son intransferibles entre consumidores y no tienen la flexibilidad del dinero. La ventaja esperable de los vales es la garantía de un mejor servicio del proveedor en competencia con otros, pues deberá aportar información adecuada a los consumidores para realizar sus elecciones de mercado, y auxiliar al gobierno como guía de consumo y redistribución del ingreso.

\subsubsection{Competencia}

La competencia cierra el cuadrángulo básico del esquema conceptual de la nueva gerencia pública, que integran la orientación al cliente, la privatización y el mercado. 
Bajo los lineamientos de la nueva gerencia pública las organizaciones de la administración pública son rediseñadas dentro de los modelos económicos del sistema de contrato, como en las empresas privadas. Es decir, se crean corporaciones competitivas que buscan buenos precios y costos bajos, de manera que estas corporaciones públicas tienen más una configuración lucrativa, que una orientación de interés público (KAUL, 1997, p. 18). Por consiguiente, la competencia es crucial porque se discierne que provee la insistencia necesaria para dar eficiencia a los servicios públicos, e incentivar a las organizaciones estatales para que sean competitivas como proveedoras del gobierno y competitivas con los proveedores del sector privado. Consiguientemente, se ha eliminado la protección monopólica que los gobiernos brindaban a sus proveedores y se han establecido servicios con base en el sistema de precios.

Debido a que la nueva gerencia pública es categorizada como una reconceptuación normativa de la administración pública, presume ser capaz de proveer servicios de alta calidad para los valores ciudadanos y el incremento de autonomía de los gerentes públicos, pues esos servicios son tan competitivos como los propios de la empresa privada (BORINS, 1995, pp. 131-132). Con base en este ideario, en el Reino Unido algunos académicos han propuesto a la nueva gerencia pública como una disciplina ecléctica y alternativa que está fundada en la economía, más que en la política, y en el énfasis en las fallas de gobierno más que en las fallas del mercado. Igualmente, han externado su escepticismo sobre la capacidad de la burocracia para proveer los servicios públicos efectivamente. Por lo tanto, la nueva gerencia pública se propone mejorar la visión de los problemas públicos del gobierno a través de fuerzas poderosas, entre las que destacan la privatización y la competitividad (BOYNE, 1996, p. 686).

La nueva gerencia pública tiene en la orientación al cliente el espacio de proximidad que requiere para poner vis a vis al proveedor y al consumidor. La privatización refrenda el papel eminente de la propiedad y el lucro que emanan del capitalismo, en tanto que el mercado se ofrece como el ámbito de intercambio y de transacción para los actores del drama económico que bulle en la administración pública cataláctica, cuya garantía es una competencia proverbial.

\subsection{APARATO DE SISTEMATIZACIÓN DE LA REFORMA NEOGERENCIAL DEL ESTADO}


El enfoque empresarial-gerencial, la gerencia por objetivos y resultados y la agenciación, configuran la base organizativa y operacional de la nueva gerencia pública.

\subsubsection{Enfoque empresarial-gerencial}

La nueva gerencia pública constituye un paradigma de gestión que combina los papeles de empresario y gerente. Entraña un modelo empresarial de manejo del sector público, pues la expresión inglesa new public management es el reflejo de la inserción de la perspectiva gerencial dentro del sector público (SAVOIE, 1995, p. 112). Esto significa la implantación de la imagen empresarial de los negocios privados dentro del gobierno, cuya idea consiste en que el sector público esté en manos de gerentes profesionales dotados con un control activo, visible y discrecional sobre las organizaciones (LANE, 1994, p. 139). Es un modelo que invoca la "libertad de manejo" como paráfrasis de la friedmaniana idea de la "libertad de elegir", de lo cual deriva su propuesta sobre la responsabilidad que requiere una asignación clara de deberes, no la dispersión del poder.

\subsubsection{Gerencia por objetivos y resultados}

El modelo neogerencial desarrolla un diseño de objetivos por resultados, donde se formulan estándares explícitos y medidas de rendimiento, así como la definición de metas e indicadores de éxito, preferentemente expresados en términos cuantitativos. La noción de responsabilidad es configurada con base en una declaración nítida de metas, de modo que se pone énfasis en los controles de resultados, en tanto que la asignación de recursos se asocia a la medida de rendimiento. Asimismo, se anteponen los resultados a los procedimientos y se enfatiza la importancia de la evaluación y la eficiencia.

\subsubsection{Agenciación}

Un propósito central del neogerencialismo es desagregar a la burocracia pública en una diversidad de agencias que operan con base en pago por servicio. La burocracia, antaño centralizada, es desarticulada en unidades menores para operar con libertad y flexibilidad. Se impone un cambio hacia la desagregación de la organización del sector público y el desgajamiento de unidades monolíticas, para establecer un sistema gerencial uniforme dentro de unidades corpóreas integradas alrededor de productos (HOOD, 1991, pp. 5-6). Las nuevas entidades operan con base en presupuestos descentralizados sobre una sola línea, de aquí que se establezcan unidades manejables merced a la separación de los intereses propios de la 
provisión y de la producción. A través de estos mecanismos se esperan ventajas derivadas del uso de contratos y franquicias, tanto dentro como fuera del sector público.

La importante noción de consumidor soberano determina directa y decisivamente las reformas de la administración pública bajo la égida neoliberal (HOOD, 1987, p. 148). La agenciación es uno de sus frutos más representativos, pues mediante esta organización en pequeña escala es como se pueden captar con mayor nitidez las preferencias del consumidor.

\subsubsection{Reducción de costos}

Como corolario de todo lo anterior, el fin supremo que se persigue consiste en disminuir los gastos del gobierno. Con miras en este objeto se acrecienta la presión hacia un gran rigor en el manejo de los recursos, la reducción de costos, el reforzamiento de la disciplina laboral y la resistencia a las demandas sindicales (HOOD, 1987, p. 146). De aquí emana el control de las demandas de recursos del sector público para "hacer más con menos". En suma: el propósito básico del gobierno neogerencial consiste en ensanchar la eficiencia en la asignación de los recursos públicos.

Buena parte de la hermandad académica neogerencial concluye lo siguiente: todo lo anterior demanda que el gobierno sea movido hacia la nueva gerencia pública, y hacia una mayor adaptación e innovación institucionales, porque aún está operando con reglas y jerarquía, limita la libertad de acción, coarta la capacidad de respuesta y restringe la iniciativa. En el gobierno la rutina es una sierva del control, cuando debiera responder al cambio con la habilidad de adaptación institucional, y así construir una más flexible y responsiva capacidad en el despacho de sus labores.

La perspectiva neogerencial involucra directamente al Estado, su gobierno y su administración, de modo que debe abandonar al modelo weberiano y adoptar el esquema de mercado. La esencia del modelo neogerencial es el neoliberalismo, cuya razón de ser consiste en haber pasado de la teoría objetiva del valor, a la teoría subjetiva del valor, que centra su atención en el individuo. Es este individuo, bautizado como consumidor, la substancia que da la vida a la nueva gerencia pública.

\section{UNA CONCLUSIÓN}

En la década de 2010 todo apuntaba al fin de la nueva gerencia pública, luego de su breve pero intensa existencia de dos décadas. Desde ese entonces, algunos académicos hicieron notar que esa gerencia había sido abordada al margen de los temas del poder, lo que 
era su pecado original, y comenzaron a tratar en su lugar el añejo tema de la gobernancia para abordar las reformas del Estado desde un ángulo diverso. Ahora, en nuestro tiempo, la nueva gerencia pública ha sido abandonada y olvidada, siendo ya, de hecho, un motivo de análisis histórico.

\section{REFERÊNCIAS}

AUCOIN, Peter. Reforma Administrativa en la Gestión Pública: Paradigmas, Principios, Paradojas y Péndulos. In: BRUGUÉ, Quim; SUBIRATS, Joan. Lecturas de Gestión Pública. Madrid: Ministerio de las Administraciones Públicas, 1996, p. 491-513.

BARZELAY, Michael. How to Argue About The New Public Management. International Public Management Journal, v. 2, n. 2, p. 183-216, 2000.

BAUDIN, Louis. L'Aube d'un nouveau libéralisme. Paris: Étions M. Th. Génin, Libraire de Médicis, 1953.

BAUDIN, Louis. EI Conflicto contemporáneo de las doctrinas económicas. México: Instituto de Investigaciones Sociales y Económicas, 1956.

BORINS, Sanford. The New Public Management is Here to Stay. Canadian Public Administration Review, v. 38, n. 1, p. 122-132, 1995.

BOYLE, Richard. Managing by Contract in the Public Sector. Administration, v. 40, n. 4, p. 333-346, 1992-1993.

BOYNE, George. The Intellectual Crisis in British Public Administration: is Public Management the Problem or the Solution?. Public Administration, v. 74, 679-694, 1996. DUNCAN, Jack; GINTER, Peter; CAPPER, STUART. Excelence in Public Administration: Four Transferable Lessons from the Private Sector. Public Productivity \& Management Review, v. 14, n. 3, p.227-236, 1991.

FRIEDMAN, Milton; FRIEDMAN, Rose. Free to choose. New York: Harcourt Brace Jovanovich, 1979.

GRAY, Andrew; JENKINS, Bill. From Public Administration to Public Management: Reassesing a Revolution. Public Administration, n. 73, p. 75-99, 1995.

GELINIER, Octave. Le Secret des structures competitives. Paris: Éditions Hommes et Techniques, 1966.

HOOD, Christopher. British Administrative Trends and the Public Choice Revolution. In: LANE, Jan-Erik (Ed). Bureaucracy and public choice. London: Sage Publications, 1987, p. 145-170. 
HOOD, Christopher. A Public Management for all Seasons?. Public Administration, n. 69, p. 3-19, 1991.

KAUL, Mohan. The New Public Administration: Management Innovations in Government.

Public Administration and Development, v. 17, n. 1, p. 13-26, 1997.

LANE, Jan-Erik. Will Public Management Drive out Public Administration. The Asian Journal of Public Administration, v. 16, n. 2, p. 139-151, 1994.

LAUFER, Romain; BURLAUD, Alain. Management public: gestion y legitimite. Paris: Delloz, 1980.

LIPPMANN, Walter. The Good society. London: George Allen \& Unwin, 1937.

LIPPMANN, Walter. La cité libre. Paris: Libraire de Médicis, 1938. [La obra fue prologada por André Maurois.]

MASSENET, Michel. La Nouvelle gestion publique: pour un Etat sans burocratie. Paris:

Editions Hommes et Tecniques, 1975.

NAVARRO, VÁZQUEZ, AGUSTÍN. Prólogo. EI Conflicto contemporáneo de las doctrinas económicas. México: Instituto de Investigaciones Sociales y Económicas, 1956. ORGANISATION FOR ECONOMIC COOPERATION AND DEVELOPMENT. The Administration as service the public as client. Paris: OECD, 1987.

ORMOND, Derry; LÖFFLER, Elke. New Public Management: What to Take and What to Leave. III International Conference of CLAD on State and Public Administration Reform, Madrid, Spain, October 14-17, 1998.

OSBORNE, David; GAEBLER, Ted. Reinventing government: how the entrepreneurial spirit is transforming the public sector. New York: Addison-Wesley, 1992.

SAVOIE, Donald. What is Wrong with the New Public Management. Canadian Public Administration Review, v. 38, n. 1, p. 112-121,1995.

PETERS, Guy; PIERRE, Jon. Citizens versus The New Public Managers: The Problem of Mutual Empowerment. Administration \& Society, United States, v. 32, n. 1, 2000 https://doi.org/10.1177/00953990022019335. 